\title{
Téoros
}

Revue de recherche en tourisme

\section{Un système informatisé dans le domaine de l'information touristique}

\section{Du rêve à la réalité ?}

\section{Pierre Bellerose}

Volume 5, numéro 3, novembre 1986

La gestion touristique : les nouvelles technologies

URI : https://id.erudit.org/iderudit/1080535ar

DOI : https://doi.org/10.7202/1080535ar

Aller au sommaire du numéro

Éditeur(s)

Université du Québec à Montréal

ISSN

0712-8657 (imprimé)

1923-2705 (numérique)

Découvrir la revue

Citer cet article

Bellerose, P. (1986). Un système informatisé dans le domaine de l'information touristique : du rêve à la réalité ? Téoros, 5(3), 17-18.

https://doi.org/10.7202/1080535ar d'utilisation que vous pouvez consulter en ligne. 


\title{
Un système informatisé dans le domaine de I'information touristique
}

\author{
par Pierre Bellerose*
}

Quel touriste ne rêve pas du jour où il pourra, du même endroit, obtenir tous les renseignements touristiques qu il desire sur le Québec grace à un certain nombre de terminaux toujours bien placés un peu partout dans la province? Et de plus, quil ne se réjouirait pas de pouvoir visionner facilement des films-vidéos sur les régions qui nous intéressent et, par après, faire une réservation soi-même parmi un vaste choix d'équipement hottelier.

Bien sür, on peut penser que tous les touristes endosseraient un tel système. Dejà on a tenté de telles expériences dans certains états américains, en Nouvelle-Écosse et dans des pays aussi éloignés que la Nouvelle-Zélande. Au Québec, certains essais isoles et plus fragmentaires ont lieu avec un certain sucoes. Ainsi, à Montréal, le système de renseignements touristiques Info-Montréal permet aux touristes d'obtenir des renseignements sur un bon nombre des activités susceptibles d'intéresser les touristes. L'expérience semble une réussite en tout point malgré un nombre restreint de terminaux disséminés dans quelques centres stratégiques de la région de Montréal.

\section{Les systèmes de réservation d'hébergement}

Depuis quelques années au Québec, il est apparu quelques systèmes isolés de réservation d'hébergement. En effet, en plus des centrales d'hébergement de certains centres de ski de l'Estrie, deux systèmes de réservation d'hébergement existent au Québec.

En effet, la chaîne hôtelière "Hôte" a développé un système maison de réservation qui semble répondre à un besoin. Les intéressés n'ont qu'à consulter le répertoire des hôteliers-membres et par la suite appeler un numéro 1-800 pour faire la réservation.

Mais c'est à Ste-Anne de Bellevue que l'on retrouve le seul système régional de réservation hôteliêre. En effet, on créait, il y a quelques mois à peine, l'organisme

"Piena Bellerose est charge de recherche en Tourisme A IUOAM.
RÉSERVOTTEL soit la Centrale de réservation en hébergement pour les régions de Québec-Charlevois. Pendant les périodes de pointes reliées à l'industrie du ski alpin, certains touristes avaient de la difficultê à effectuer des réservations dans la région de Québec. Selon Daniel Bouchard de Réservôtel, la decision de mettre sur pied un tel système a êté notivés par la décision de l'Office du Tourisme et des Congrès de Québec de sabrer dans les budgets des lignes 1-800 qui servaient aux réservations. Actuellement, Réservôtel comprend 71 hoteliers de Charlevoix et de la grande région de Québec qui paient une commission pour l'autofinancement de ce système et l'expérience jusqu'ici, semble avoir un succès qui dépasse les objectifts des organis ateurs.

\section{Un projet ambitient émanant du Ministère du Tourisme}

Et maintenant, à Québec, quelques fonctionnaires "mijotent" depuis déjà près de trois ans un projet ambitieux de système informatisé de réservation d'hébergement et de renseignement touristique. Le projet qui a été déposé au Conseil Québécois du Tourisme en janvier 1986, devrait être présenté au Conseil des ministres avant la fin de l'année pour approbation finale. Il s'agit d'un projet échelonné sur 3 ans, de plus de $\$ 18$ millions et qui a comme objectif principal de "supporter dans la poursuite de leurs objectifs les principaux intervenants de l'industrie touristique québécoise en mettant à leur disposition, contre rémuneration, un ensemble intégré de systêmes informatisés efficaces, tels ceux de réservation d'hébergement et de renseignement" ". Le Ministère du Tourisme, pour arriver a ces buts, mettrait sur pied une société à but non lucratif de type privé qui devrait atteindre l'autofinancement une fois les investissements accomplis.

Evidemment, on espere avec ce systeme augmenter la demande touristique (hausse du nombre de visiteurs au Québec, meilleure rétention au Québec des québécois prenant habituellement leurs vacances à l'extérieur et enfin prolongation des visites des touristes deja sur place) mais aussi assurer une meilleure distribution régionale

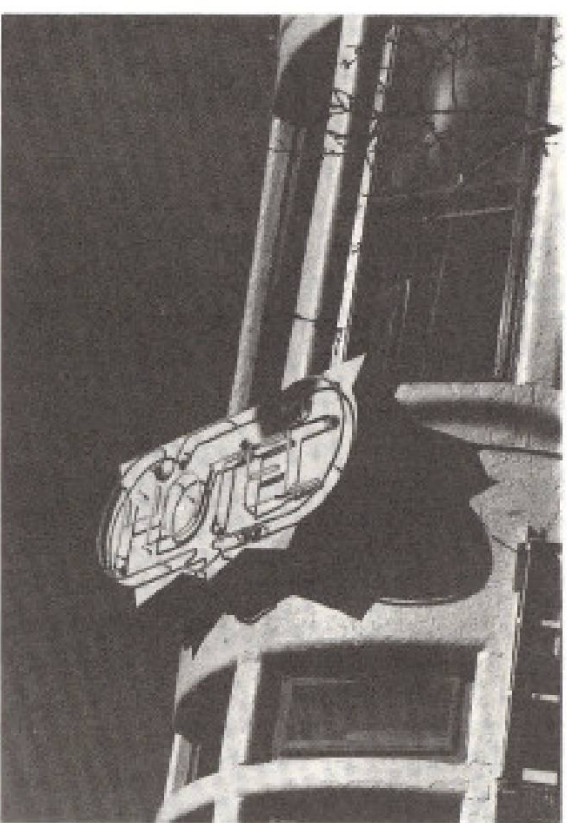

Des rêservations en un clin d'oeil

de l'activité touristique. Il faut ajouter que face à la concurrence internationale, le Québec peut perdre une part de son marché s'il ne s"équipe pas des outils nécessaires à son développement.

\section{Un système intégré}

En août 1984, le Gouvernement du Québec mandatait plusieurs firmes de consultants pour étudier la structure, les modes de financement ainsi que les modalités d'application de ce système. Malheureusement, même si les résultats de ces travaux sont dếposés depuis 1 an au Ministere du Tourisme, il est impossible de les consulter puisque tout le dossier est à l'étude au Conseil du Trésor. Toutefois, nous pouvons présenter les grandes lignes du projet grâce aux informations obtenues de Renaud Plamondon du Ministere du Tourisme à Québec.

Ainsi, si le projet est accepté par le Conseil des ministres, la première année on s'en tiendrait à la création d'une banque de données qui sera utilisće uniquement pour l'usage des préposés à l'accucil. Ce sera la base informatique nécessaire à la création du système de renseignement. Par la suite, 


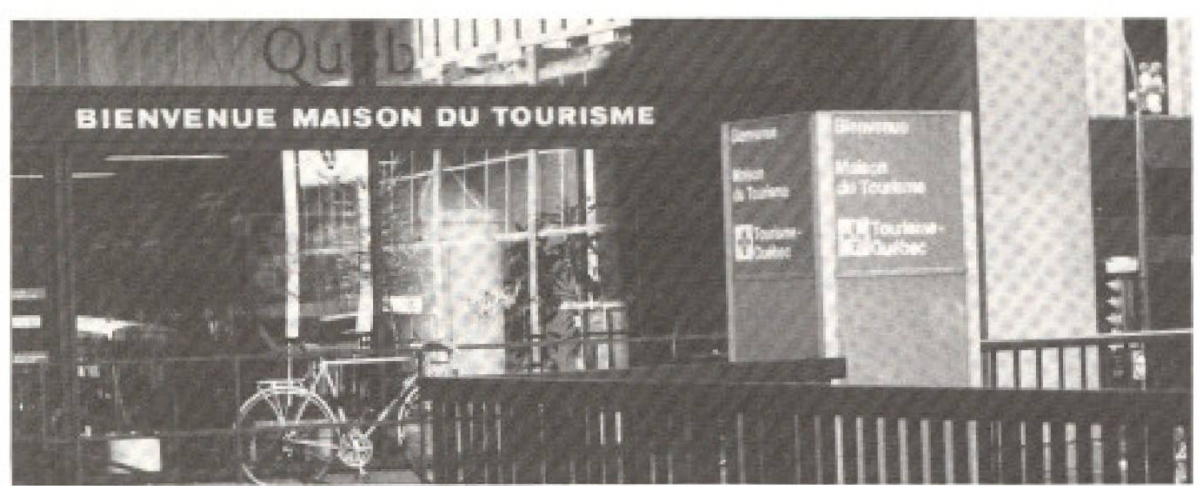

Vers un système intégré d'information

au cours des deux années suivantes, on mettra en place graduellement les autres composantes du système intégré de renseignement et de réservation d'hébergement.

Comme on le voit au tableau 1 , les utilisateurs de ce système seront bien sûr le grand public et les préposés au renseignement mais aussi certains échanges pourtaient être faits avec d'autres systèmes de réservation.

Le système, d'unc façon indirecte, permettra au Ministère du Tourisme d'obtenir de meilleures données sur les tendances du marché, ce qui améliorera la recherche dans ce domaine. Pour ce qui est du système de réservation prévu dans ce projet, à peu pres tous ces équipements d'hébergement seront intégrés dans le réseau (même les équipements relevant du M.L.C.P.). Cependant, puisqu'on prévoit un autofinancement des opérations, on doit donc supposer que les offreurs de services devront payer une commission.

On peut donc se demander si tous les participants seront sur le même pied. Est-ce que ce ne seront pas encore une fois les plus importants propriétaires d'équipements hôteliers qui profiteront de ces nouvelles structures au détriment des petits hôteliers.

\section{Tableau I}

Présentation de façon
systématique du projet
de réservation
d'hébergement
et de renseignement
touristique

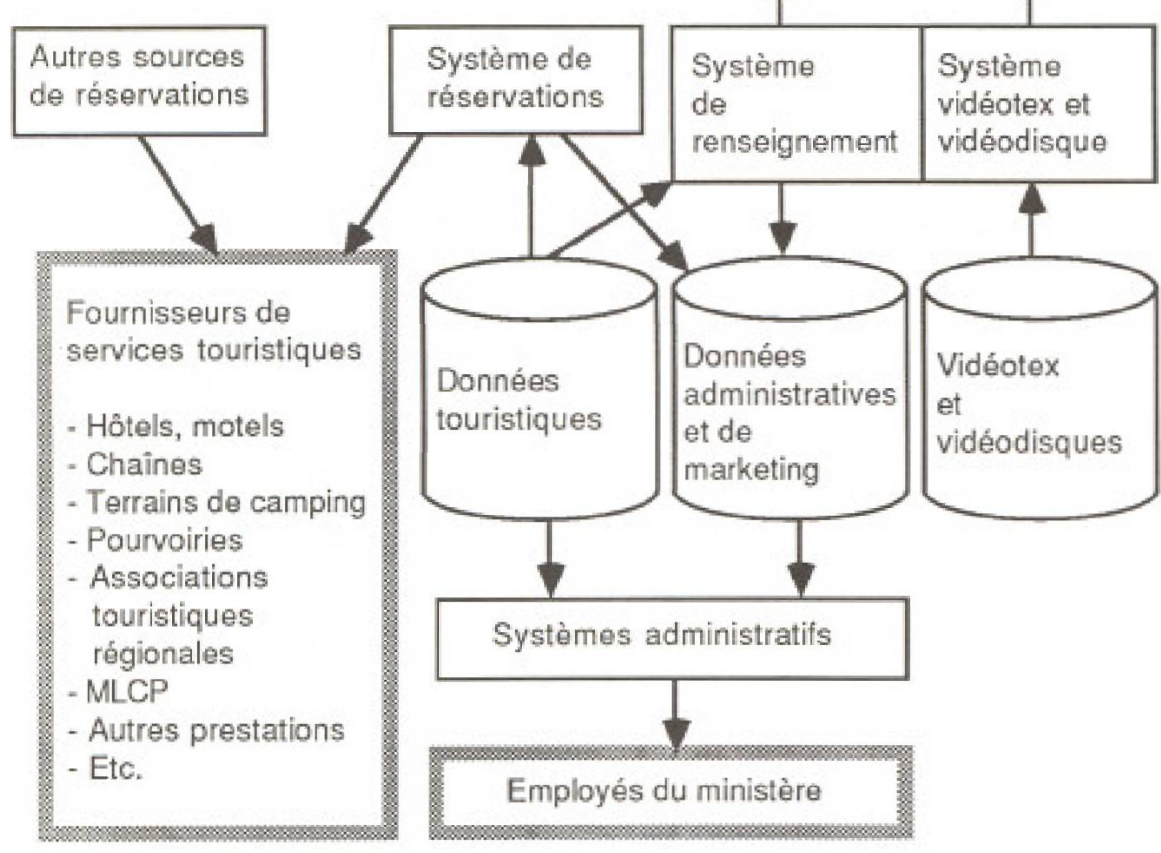

des aubergistes ou des gestionnaires d'équipements de tourisme social qui n'auront peut-être pas les moyens de défrayer les coûts inhérents à un tel système? On ne connaîtra pas les modalités du système avant quelques temps, mais on espere que les administrateurs publics tiendront compte de l'accessibilité à ce service pour le milieu touristique, sinon tout le projet risque de perdre de sa crédibilité.

Pour ce qui est des renseignements présentés aux touristes, ils seront véhiculés à l'aide de supports se conformant a la fine pointe de la technologie. Ainsi, on ne s'en tiendra pas uniquement aux pages informatiscés (vidéotextes à l'exemple d'InfoMontréal) transmises à l'aide de terminaux mais, en plus, on prévoit offrir aux touristes des courts vidéos (vidéodisques) sur les principales régions touristiques er même éventuellement sur certaines attractions.

Plus tard, on espère installer certains équipements périphériques tels une imprimante et un lecteur de carte de crédit. Bien sûr, toutes ces composantes seront implantées avec l'objectif d'augmenter la demande pour les régions touristiques du Québec.

Un tel projet à incidence technologique, $s$ 'il est accepté, changera grandement le visage de l'industrie touristique et surtout la perception que s'en fera le touriste. Les intervenants de tous les secteurs devront vivre, et le mieux possible, avec ces nouveaux outils.

Cependant, malgré tous les avantages évidents que l'on retrouve dans le système proposé, on doit se demander si on ne devrait pas utiliser les $\$ 18$ millions dans certaines réalisations plus urgentes. Ne devrait-on pas plutôt investir dans le produit touristi= que québécois et sa promotion plutôt que dans un tel projet? A quoi bon investir dans un système de renseignement et de réservation hyper-sophistiqué si le produit n'est pas à la hauteur?

Quoi qu'il en soit, d'ici quelques semaines, le Conseil des ministres statuera sur la pertinence de ce projet et plusieurs intervenants sauront alors si toutes ces rêgles du jeu seront changées. C'est à suivre... f

\begin{abstract}
Remarcinments
J'aimerais remercier pour leurs collaborations a cet sarticle: Chantale Tessier, eftudiante en tourisme a l'UOAMLouis Jolin, professeur au Module de gestion et inter vention touristiquas; Renaud Plamondon du Ministére du Tourisme du Québec; Pierre Labrie de CIDEMTourisme: Michêle Lortie de la Socièté du Palais dess Congrès; Daniel Bouchard de Réservōtel et enfin Suzanne Jacques de l'agence de voyage Fleur de Lys.
\end{abstract}

\section{Béférence}

Ministere du Tourisme, Système informatise de réservation d'hébergement et de renseignement touristique. Document présenté au Conseil Québécois du Tourisme, le 17 janvier 1986. 